\title{
Nonlinear observer for hydropower system
}

\author{
Liubomyr Vytvytskyi Roshan Sharma Bernt Lie
}

Department of Electrical engineering, Information Technology and Cybernetics, University of South-Eastern Norway, Porsgrunn, Norway. E-mail: \{Liubomyr.Vytvytskyi, Roshan.Sharma,Bernt.Lie\} @usn.no

\begin{abstract}
Estimation of unmeasured states plays an essential role in the design of control systems as well as for monitoring of hydropower plants. The standard Kalman filter gives the optimum state estimates for linear systems. However, this optimality is not relevant for nonlinear models and a choice between stochastic and deterministic approaches is not so obvious in this case. Thus the application of a nonlinear observer in a hydropower system is of interest here as an alternative to the widely used extended Kalman filter.

This paper provides a study and design of a reduced order nonlinear observer to estimate the states of a hydropower system. Implementation of the nonlinear observer is done in OpenModelica and added to our in-house hydropower Modelica library — OpenHPL, where different models for hydropower systems are assembled. Simulations and analysis of the designed observer are done in Python using a Python API for operating OpenModelica simulations.
\end{abstract}

Keywords: State estimation, reduced order nonlinear observer, hydropower model

\section{Introduction}

\subsection{Background}

A transition towards more renewable energy sources is currently happening in Europe and all over the world. This situation leads to increased use of flexible hydropower plants to compensate for the highly changing production from intermittent energy sources such as wind and solar irradiation. For this reason, development of advanced control structures or optimization of existing controllers for hydropower plants become a key task.

Full state information of the model of a hydropower system is needed to design an advanced controller such as model predictive control (MPC) or other model based controllers. However, some of the states can not be directly measured. Instead, a combination of a mathematical model and available measurements can be used to estimate the unmeasured states in hydropower plants.

Popular state estimation methods include the Kalman filter (KF) with a wide range of extensions that apply depending on model structure, computational effort, etc. The Kalman filter is based on a stochastic approach. Alternatively, an observer based on a deterministic approach can be used for state estimation. This alternative is of particular interest for nonlinear systems such as the hydropower one, because the standard Kalman filter provides an optimum solution only for linear system, Anderson and Moore (1979). In addition, a nonlinear observer can lead to significant reduction of computational effort compared to a Kalman filter. Such observers can quickly find an estimate of the states and provide these as an input to fast nonlinear control algorithms. On the other hand, the proof of convergence is non-trivial for nonlinear observers.

\subsection{Previous Work}

A basic introduction to a variety of state estimation techniques based on the stochastic approach for general linear and nonlinear systems is provided in Simon (2006). As an alternative, an observer based on a deterministic approach can be used for the state estimation 
and was first proposed by Luenberger (1964) for linear systems. Extension of the linear observer design for nonlinear systems has been presented in Krener and Isidori (1983). Although a huge variety of techniques for designing nonlinear observer exist, Kravaris et al. (2007); El-Farra et al. (2005); Andrieu and Praly (2006), the design of nonlinear observers is still a challenging task.

Some work on modeling the waterway for a high head hydropower system together with a generator, the Francis turbine, and a governor, using OpenModelica ${ }^{1}$, is given in Vytvytskyi and Lie (2017, 2018b). Unit models have been assembled in our in-house Modelica ${ }^{2}$ library OpenHPL.

A Python $\mathrm{API}^{3}$ for OpenModelica already exists and provides the possibilities for performing simulations of OpenModelica models via Python ${ }^{4}$, Lie et al. (2016). Python in turn gives much wider possibilities for plotting, analysis, and optimization than what is possible in OpenModelica Vytvytskyi and Lie (2018a).

\subsection{Overview of Paper}

In this paper, the main contribution is the development and simulation study of a reduced order nonlinear observer for state estimation in a hydropower system. Implementation of the observer is done in OpenModelica using OpenHPL. Simulation and analysis of the designed observer is done in Python using the Python API for OpenModelica.

The paper is structured as follows: Section 2 gives a system description of a high head hydropower plant. Section 3 gives an overview of the hydropower model. Design and proof of convergence for the nonlinear reduced order observer for the hydropower system is provided in Section 4. Simulation results from a number of case studies are presented in Sections 5 and 6. Finally, discussion and conclusions are given in Section 7.

\section{System description}

High head plants typically collect and store water in reservoirs in mountains, with tunnels leading the relatively small flow of water down a considerable height difference to the aggregated turbine and generator. The electricity produced by the generator is then transferred through power lines to consumers. A typical structure for a high head hydropower plant is depicted in Fig. 1, Vytvytskyi and Lie (2017).

\footnotetext{
$1_{\text {https: //openmodelica.org }}$

2 https://www.modelica.org

${ }^{3}$ https://goo.gl/Qyjqq2

4 https://www.python.org
}

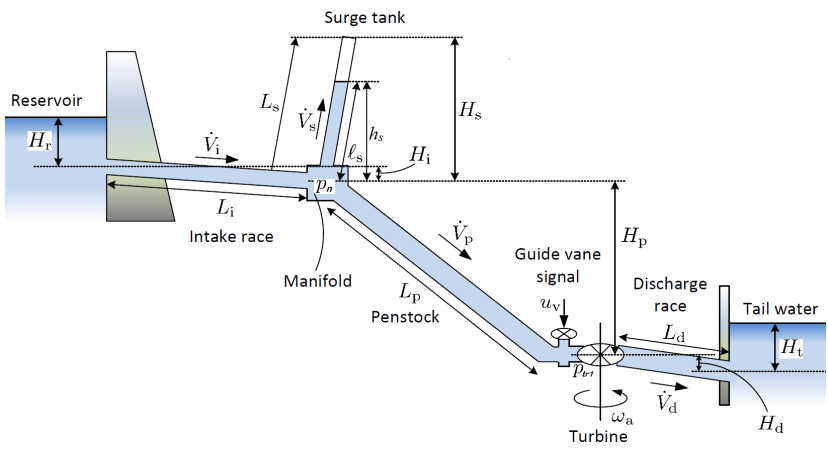

Figure 1: Overview of the structure of the high head hydropower plant.

For simulations in this paper, data from the Sundsbarm hydropower plant in Telemark, Norway is used with data provided in Vytvytskyi and Lie (2017).

\section{Model}

\subsection{Model overview}

For modeling the hydropower system, Modelica library OpenHPL is used. This is an in-house hydropower library, where different parts of the waterway components such as reservoir, conduit, surge tank, and turbine, have been assembled. In this library, different waterway components of the hydropower system are described by both mass and momentum balance, and include compressible/incompressible water or elastic/inelastic pipe walls. An overview of the mathematical models and methods used in this library is given in Vytvytskyi and Lie (2017); Splavska et al. (2017).

In this study, a simple hydropower model for the waterway is considered with the following assumptions for simplification:

- constant water level in reservoirs,

- power generation simplifications (constant speed of turbine),

- incompressible water and inelastic pipe.

All these simplifications lead to a simple hydropower model that consists of only three states Vytvytskyi and Lie (2018a).

\subsection{Model presentation}

In Modelica, models are described as differential algebraic equations (DAEs), with differential and algebraic variables. OpenModelica by default transforms the DAEs into state space form with auxiliary variables: states are typically a subset of the differential 
variables, while the auxiliary variables are the remaining variables. For the design of nonlinear observers, the model of the hydropower plant is represented by ordinary differential equations (ODE). The states of the model are given in vector $x$ as follows:

$$
x=\left[\begin{array}{c}
\dot{V}_{\mathrm{p}} \\
\dot{V}_{\mathrm{s}} \\
h_{\mathrm{s}}
\end{array}\right]=\left[\begin{array}{c}
x_{1} \\
x_{2} \\
x_{3}
\end{array}\right]
$$

Here, $\dot{V}_{\mathrm{p}}$ is the volumetric flow rate through the penstock (equal to the flow rate in the discharge), $\dot{V}_{\mathrm{S}}$ is the volumetric flow rate in the surge tank, and $h_{\mathrm{s}}$ is the level in the surge tank, see Fig. 1. The sum of the flow rates in the penstock and the surge tank defines the flow rate in the intake: $\dot{V}_{\mathrm{i}}=x_{1}+x_{2}$. The states are described by differential equations as follows:

$$
\begin{aligned}
& \frac{d x_{1}}{d t}=\frac{Z_{\mathrm{p}}}{\rho}\left(p_{\mathrm{n}}-p_{\mathrm{tr} 1}\right)+A_{\mathrm{p}} g \frac{H_{\mathrm{p}}}{L_{\mathrm{p}}} \\
& -\frac{1}{8} \pi f_{\mathrm{D}} B_{\mathrm{p}} x_{1}\left|x_{1}\right| \\
& \frac{d x_{2}}{d t}=\frac{Z_{\mathrm{s}}}{\rho}\left(p_{\mathrm{n}}-p^{\mathrm{atm}}\right)-A_{\mathrm{s}} g \frac{H_{\mathrm{s}}}{L_{\mathrm{s}}} \\
& -\frac{1}{8} \pi f_{\mathrm{D}} B_{\mathrm{s}} x_{2}\left|x_{2}\right| \\
& \frac{d x_{3}}{d t}=\frac{x_{2} H_{\mathrm{s}}}{A_{\mathrm{s}} L_{\mathrm{s}}}
\end{aligned}
$$

Here, $p_{\mathrm{n}}$ is the manifold node pressure, $p_{\mathrm{tr} 1}$ is the turbine inlet pressure, $p^{\text {atm }}$ is the atmospheric pressure. $A_{\mathrm{p}}$ and $A_{\mathrm{s}}$ are the cross section areas of the penstock and surge tank, respectively. $H_{\mathrm{p}}, H_{\mathrm{s}}$, and $L_{\mathrm{p}}, L_{\mathrm{s}}$ are the height differences and the lengths of the penstock and surge tank, respectively. $g$ is the gravitational acceleration and $\rho$ is the water density. $f_{\mathrm{D}}$ is the Darcy friction factor and is assumed to be constant for simplicity. $B_{\mathrm{p}}, B_{\mathrm{s}}, Z_{\mathrm{p}}$ are geometrical parameters of the penstock and surge tank, and are presented in Eq. 5 and 6.

$$
\begin{gathered}
Z_{\mathrm{s}}=\frac{A_{\mathrm{s}} H_{\mathrm{s}}}{x_{3} L_{\mathrm{s}}}, \quad Z_{\mathrm{i}}=\frac{A_{\mathrm{i}}}{L_{\mathrm{i}}}, \quad Z_{\mathrm{p}}=\frac{A_{\mathrm{p}}}{L_{\mathrm{p}}}, \quad Z_{\mathrm{d}}=\frac{A_{\mathrm{d}}}{L_{\mathrm{d}}} \\
B_{\mathrm{s}}=\frac{D_{\mathrm{s}}}{A_{\mathrm{s}}^{2}}, \quad B_{\mathrm{i}}=\frac{D_{\mathrm{i}}}{A_{\mathrm{i}}^{2}}, \quad B_{\mathrm{p}}=\frac{D_{\mathrm{p}}}{A_{\mathrm{p}}^{2}}, \quad B_{\mathrm{d}}=\frac{D_{\mathrm{d}}}{A_{\mathrm{d}}^{2}}
\end{gathered}
$$

Here, $Z_{\mathrm{p}}, Z_{\mathrm{s}}, Z_{\mathrm{d}}, Z_{\mathrm{i}}$ are ratios of the cross section area to the length of the penstock, surge tank, discharge, and intake, respectively. $B_{\mathrm{p}}, B_{\mathrm{s}}, B_{\mathrm{d}}, B_{\mathrm{i}}$ are also ratios of the diameter to the squared cross section area of the penstock, surge tank, discharge, and intake, respectively.

The manifold and turbine inlet pressures are defined as follows:

$$
\begin{aligned}
& p_{\text {tr1 }}=K_{\mathrm{z}}\left[Z_{\mathrm{p}} p_{\mathrm{n}}+\rho g\left(A_{\mathrm{p}} \frac{H_{\mathrm{p}}}{L_{\mathrm{p}}}-A_{\mathrm{d}} \frac{H_{\mathrm{d}}}{L_{\mathrm{d}}}\right)\right. \\
& +Z_{\mathrm{d}}\left(\frac{x_{1}^{2} p^{\mathrm{atm}}}{C_{\mathrm{v}}^{2} u_{\mathrm{v}}^{2}}+p_{\mathrm{t}}\right) \\
& \left.+\frac{1}{8} \pi \rho x_{1}\left|x_{1}\right|\left(f_{\mathrm{D}, \mathrm{d}} B_{\mathrm{d}}-f_{\mathrm{D}, \mathrm{p}} B_{\mathrm{p}}\right)\right] \\
& p_{\mathrm{n}}=K_{p}\left[Z_{\mathrm{i}} p_{\mathrm{r}}+Z_{\mathrm{s}} p^{\mathrm{atm}}+K_{\mathrm{pdz}}\left(\frac{x_{1}^{2} p^{\mathrm{atm}}}{C_{\mathrm{v}}^{2} u_{\mathrm{v}}^{2}}+p_{\mathrm{t}}\right)\right. \\
& +\rho g\left(A_{\mathrm{p}} \frac{H_{\mathrm{p}}}{L_{\mathrm{p}}}\left(K_{\mathrm{pd}}-1\right)-K_{\mathrm{pd}} A_{\mathrm{d}} \frac{H_{\mathrm{d}}}{L_{\mathrm{d}}}+A_{\mathrm{s}} \frac{H_{\mathrm{s}}}{L_{\mathrm{s}}}\right. \\
& \left.+A_{\mathrm{i}} \frac{H_{i}}{L_{i}}\right)+\frac{1}{8} \pi \rho\left(f_{\mathrm{D}, \mathrm{s}} B_{\mathrm{s}} x_{2}\left|x_{2}\right|\right. \\
& -f_{\mathrm{D}, \mathrm{i}} B_{\mathrm{i}}\left(x_{2}+x_{1}\right)\left|x_{2}+x_{1}\right| \\
& \left.\left.+\left(f_{\mathrm{D}, \mathrm{d}} B_{\mathrm{d}} K_{\mathrm{pd}}+f_{\mathrm{D}, \mathrm{p}} B_{\mathrm{p}}\left(1-K_{\mathrm{pd}}\right)\right) x_{1}\left|x_{1}\right|\right)\right]
\end{aligned}
$$

Here, $C_{\mathrm{v}}$ is the turbine valve capacity and $u_{\mathrm{v}}$ is the turbine valve signal. $p_{\mathrm{r}}$ and $p_{\mathrm{t}}$ are the inlet penstock and the outlet discharge pressures, respectively. These pressures are defined from the atmospheric pressure and depths of the reservoir $-H_{\mathrm{r}}$, and tail water $-H_{\mathrm{t}}$ as follows:

$$
\begin{aligned}
& p_{\mathrm{r}}=p^{\mathrm{atm}}+\rho g H_{\mathrm{r}} \\
& p_{\mathrm{t}}=p^{\mathrm{atm}}+\rho g H_{\mathrm{t}}
\end{aligned}
$$

Coefficients $K_{\mathrm{z}}, K_{\mathrm{pd}}, K_{\mathrm{pdz}}$, and $K_{p}$ are defined from the geometric ratios $Z$ as follows:

$$
\begin{aligned}
& K_{\mathrm{z}}=\frac{1}{Z_{\mathrm{d}}+Z_{\mathrm{p}}}, \quad K_{\mathrm{pdz}}=Z_{\mathrm{p}} Z_{\mathrm{d}} K_{\mathrm{z}} \\
& K_{\mathrm{pd}}=Z_{\mathrm{p}} K_{\mathrm{z}}, \quad K_{p}=\frac{1}{Z_{\mathrm{i}}+Z_{\mathrm{s}}+K_{\mathrm{pdz}}}
\end{aligned}
$$

This simple hydropower model is next used to design the reduced order nonlinear observer.

\section{Observer design}

Consider a general nonlinear system with state $x$, control signal $u$, output $y$ and nonlinear functions $f()$ and $g()$ for the state and output, respectively:

$$
\begin{aligned}
& \frac{d x}{d t}=f(x, u) \\
& y=g(x, u)
\end{aligned}
$$


A standard presentation of any full order observer is given by Eq. 12,

$$
\begin{gathered}
\frac{d \hat{x}}{d t}=f(\hat{x}, u)+L(y-\hat{y}) \\
\hat{y}=g(\hat{x}, u)
\end{gathered}
$$

Here, $\hat{x}$ and $\hat{y}$ are the estimates of state $x$ and output $y$, respectively. $L$ is the observer gain and could be a nonlinear function, $L=L(x, u)$. The observation error dynamic, $\tilde{x}$, is described as:

$$
\frac{d \tilde{x}}{d t}=\frac{d x}{d t}-\frac{d \hat{x}}{d t}=f(x, u)-f(\hat{x}, u)-L(y-\hat{y})
$$

The observer gain should be chosen or designed such that $\frac{d \tilde{x}}{d t}$ is an asymptotically stable system. This design can be done using Lyapunov based nonlinear system stability analysis, Smith (1995), and is, in general, a challenging task.

In real hydropower plants, the states $x_{1}$ and $x_{3}$ are usually available as measurements. Thus, in this study these two states are assumed to be known. The unknown state is $x_{2}$. A reduced order observer for estimating the unmeasured state $x_{2}$ is designed for the hydropower system. The following variable transformation is chosen for observing $x_{2}$ :

$$
\zeta_{2}=x_{2}+L_{1} x_{1}+L_{3} x_{3}
$$

Here, $L_{1}$ and $L_{3}$ are the observer gains, also known as injection gains. Injection from both measured states $x_{1}$ and $x_{3}$ are used to design the nonlinear observer. However, the discussion on the estimation of $x_{2}$ by injecting only one of the measured states is provided in Section 5. From Eq. 14, the dynamics for $\zeta_{2}$ is,

$$
\frac{d \zeta_{2}}{d t}=\frac{d x_{2}}{d t}+L_{1} \frac{d x_{1}}{d t}+L_{3} \frac{d x_{3}}{d t}
$$

The dynamics of the measured states $x_{1}$ and $x_{3}$ from Eq. 2 and 4 are inserted into Eq. 15 .

$$
\begin{aligned}
\frac{d \zeta_{2}}{d t}=\frac{Z_{\mathrm{s}}}{\rho}\left(p_{\mathrm{n}}\right. & \left.-p^{\mathrm{atm}}\right)-A_{\mathrm{s}} g \frac{H_{\mathrm{s}}}{L_{\mathrm{s}}} \\
-\frac{1}{8} \pi & f_{\mathrm{D}, \mathrm{s}} B_{\mathrm{s}} x_{2}\left|x_{2}\right|+L_{3}\left(\frac{x_{2} H_{\mathrm{s}}}{A_{\mathrm{s}} L_{\mathrm{s}}}\right) \\
& +L_{1}\left(\frac{Z_{\mathrm{p}}}{\rho}\left(p_{\mathrm{n}}-p_{\mathrm{tr} 1}\right)\right. \\
& \left.+A_{\mathrm{p}} g \frac{H_{\mathrm{p}}}{L_{\mathrm{p}}}-\frac{1}{8} \pi f_{\mathrm{D}, \mathrm{p}} B_{\mathrm{p}} x_{1}\left|x_{1}\right|\right)
\end{aligned}
$$

Now, the observer for $x_{2}$ is,

$$
\begin{aligned}
\frac{d \hat{\zeta}_{2}}{d t}=\frac{Z_{\mathrm{s}}}{\rho}\left(p_{\mathrm{n}}\right. & \left.-p^{\mathrm{atm}}\right)-A_{\mathrm{s}} g \frac{H_{\mathrm{s}}}{L_{\mathrm{s}}} \\
& -\frac{1}{8} \pi f_{\mathrm{D}, \mathrm{s}} B_{\mathrm{s}} \hat{x}_{2}\left|\hat{x}_{2}\right|+L_{3}\left(\frac{\hat{x}_{2} H_{\mathrm{s}}}{A_{\mathrm{s}} L_{\mathrm{s}}}\right) \\
& +L_{1}\left(\frac{Z_{\mathrm{p}}}{\rho}\left(p_{\mathrm{n}}-p_{\mathrm{tr} 1}\right)\right. \\
& \left.+A_{\mathrm{p}} g \frac{H_{\mathrm{p}}}{L_{\mathrm{p}}}-\frac{1}{8} \pi f_{\mathrm{D}, \mathrm{p}} B_{\mathrm{p}} x_{1}\left|x_{1}\right|\right)
\end{aligned}
$$

The estimation error dynamic is defined as:

$$
\frac{d \tilde{\zeta}_{2}}{d t} \equiv \frac{d \zeta_{2}}{d t}-\frac{d \hat{\zeta}_{2}}{d t}
$$

After inserting $\frac{d \zeta_{2}}{d t}$ and $\frac{d \hat{\zeta}_{2}}{d t}$ from Eq. 16 and 17 into Eq. 18, the estimation error dynamic is,

$$
\begin{aligned}
& \frac{d \tilde{\zeta}_{2}}{d t}=\frac{1}{8} \pi f_{\mathrm{D}, \mathrm{s}} B_{\mathrm{s}}\left[\left(Z_{\mathrm{s}}+L_{1} K_{\mathrm{pdz}}\right) K_{p}-1\right] \\
& \times\left(x_{2}\left|x_{2}\right|-\hat{x}_{2}\left|\hat{x}_{2}\right|\right)+\frac{L_{3} H_{\mathrm{s}}}{A_{\mathrm{s}} L_{\mathrm{s}}} \tilde{\zeta}_{2} \\
& \quad-\frac{1}{8} \pi\left(Z_{\mathrm{s}}+L_{1} K_{\mathrm{pdz}}\right) K_{p} f_{\mathrm{D}, \mathrm{i}} B_{\mathrm{i}} \\
& \times\left(\left(x_{2}+x_{1}\right)\left|x_{2}+x_{1}\right|-\left(\hat{x}_{2}+x_{1}\right)\left|\hat{x}_{2}+x_{1}\right|\right)
\end{aligned}
$$

The estimation error dynamic should be analyzed for stability so that its state converges to zero in a finite amount of time. For this reason, Lyapunov stability analysis is used and a candidate Lyapunov function $V$ is considered as follows:

$$
V=\frac{1}{2} \tilde{\zeta}_{2}^{2}
$$

For the Lyapunov analysis, it must be shown that a derivative of the Lyapunov function is less than or equal to zero: $\frac{d V}{d t} \leq 0$. So, the derivative of this Lyapunov function is,

$$
\frac{d V}{d t}=\tilde{\zeta}_{2} \frac{d \tilde{\zeta}_{2}}{d t}
$$

Inserting the observation error dynamic from Eq. 19, the derivative of the Lyapunov function $\frac{d V}{d t}$ becomes as follows: 


$$
\begin{gathered}
\frac{d V}{d t}=\frac{1}{8} \pi f_{\mathrm{D}, \mathrm{s}} B_{\mathrm{s}}\left[\left(Z_{\mathrm{s}}+L_{1} K_{\mathrm{pdz}}\right) K_{p}-1\right] \\
\times\left(x_{2}\left|x_{2}\right|-\hat{x}_{2}\left|\hat{x}_{2}\right|\right) \tilde{\zeta}_{2}+\frac{L_{3} H_{\mathrm{s}}}{A_{\mathrm{s}} L_{\mathrm{s}}} \tilde{\zeta}_{2}^{2} \\
\quad-\frac{1}{8} \pi\left(Z_{\mathrm{s}}+L_{1} K_{\mathrm{pdz}}\right) K_{p} f_{\mathrm{D}, \mathrm{i}} B_{\mathrm{i}} \\
\times\left(\left(x_{2}+x_{1}\right)\left|x_{2}+x_{1}\right|-\left(\hat{x}_{2}+x_{1}\right)\left|\hat{x}_{2}+x_{1}\right|\right) \tilde{\zeta}_{2}
\end{gathered}
$$

Equation 22 has three terms on its right hand side. Each of these terms will be analyzed separately and it will be shown that each term fulfills the conditions for Lyapunov stability. In the process of analyzing Eq. 22, the conditions for observer gains $L_{1}$ and $L_{3}$ will be calculated. From the first term on the right hand side of Eq. 22, let us suppose,

$$
f_{2}=\left(x_{2}\left|x_{2}\right|-\hat{x}_{2}\left|\hat{x}_{2}\right|\right)\left(x_{2}-\hat{x}_{2}\right)
$$

Then, the following conditions exist:

$$
\left\{\begin{array}{l}
\text { if } \quad x_{2}-\hat{x}_{2}=\tilde{\zeta}_{2}=0, \quad \text { then } \quad f_{2}=0 \\
\text { if } \quad x_{2}-\hat{x}_{2}=\tilde{\zeta}_{2} \neq 0, \quad \text { then } f_{2}>0
\end{array}\right.
$$

Hence, $f_{2} \geq 0$, i.e., $f_{2}$ is positive semidefinite. This implies that,

$$
\frac{1}{8} \pi f_{\mathrm{D}, \mathrm{s}} B_{\mathrm{s}}\left[\left(Z_{\mathrm{s}}+L_{1} K_{\mathrm{pdz}}\right) K_{p}-1\right] \leq 0
$$

From Equation 25, a condition for the observer gain $L_{1}$ can be defined as follows:

$$
L_{1} \geq \frac{Z_{\mathrm{s}}}{K_{\mathrm{pdz}}}-\frac{1}{K_{\mathrm{pdz}} K_{p}}
$$

Next, the second term on the right hand side of Eq. 22 can be analyzed. Here, it is obvious that $\tilde{\zeta}_{2}^{2} \geq 0$. For this second term to be negative semi definite, the condition given by Eq. 27 should be fulfilled. Thus, the condition for the observer gain $L_{3}$ is,

$$
\frac{L_{3} H_{\mathrm{s}}}{A_{\mathrm{s}} L_{\mathrm{s}}} \leq 0 \rightarrow L_{3} \leq 0
$$

Finally, from the third term on the right hand side of Eq. 22, let us suppose,

$$
\begin{aligned}
& f_{1,2}=\left(\left(x_{2}+x_{1}\right)\left|x_{2}+x_{1}\right|\right. \\
&\left.-\left(\hat{x}_{2}+x_{1}\right)\left|\hat{x}_{2}+x_{1}\right|\right) \times\left(x_{2}-\hat{x}_{2}\right)
\end{aligned}
$$

Knowing that $x_{1} \geq 0$ (flow rate in the penstock is positive), it follows that,

$$
\left\{\begin{array}{lll}
\text { if } \quad x_{2}-\hat{x}_{2}=\tilde{\zeta}_{2}=0, & \text { then } \quad f_{1,2}=0 \\
\text { if } \quad x_{2}-\hat{x}_{2}=\tilde{\zeta}_{2} \neq 0, & \text { then } \quad f_{1,2}>0
\end{array}\right.
$$

Hence, $f_{1,2} \geq 0$, i.e., $f_{1,2}$ is positive semidefinite. This implies that,

$$
\frac{1}{8} \pi\left(Z_{\mathrm{s}}+L_{1} K_{\mathrm{pdz}}\right) K_{p} f_{\mathrm{D}, \mathrm{i}} B_{\mathrm{i}} \geq 0
$$

From here, another condition for the observer gain $L_{1}$ is found,

$$
L_{1} \leq \frac{Z_{\mathrm{s}}}{K_{\mathrm{pdz}}}
$$

Thus from Eq. 26, 27 and 31, the required condition for the stable dynamics of the estimation error dynamic $\frac{d \tilde{\zeta}_{2}}{d t}$ is:

$$
\begin{gathered}
\frac{Z_{\mathrm{s}}}{K_{\mathrm{pdz}}}-\frac{1}{K_{\mathrm{pdz}} K_{p}} \leq L_{1} \leq \frac{Z_{\mathrm{s}}}{K_{\mathrm{pdz}}} \\
L_{3} \leq 0
\end{gathered}
$$

Hence, the designed reduced order nonlinear observer for estimating the volumetric flow rate in the surge tank $\left(x_{2}\right)$ is written as,

$$
\hat{x}_{2}=\hat{\zeta}_{2}-L_{1} x_{1}-L_{3} x_{3}
$$

Here, $\hat{\zeta}_{2}$ is the estimate of the transformed coordinate for $x_{2}$ and its dynamics is defined using Eq. 17. $L_{1}$ and $L_{3}$ are the observer gains that are defined using conditions in Eq. 32. A summary of the developed reduced order nonlinear observer for the hydropower system is given in Table 1 .

Thus, the observer convergence is proved here, and this in turn proves observability of the state $x_{2}$. Then, the observer simulations are illustrated in the next section.

\section{Results and discussions}

\subsection{Simulation setup}

The reduced order nonlinear observer is implemented in OpenModelica, which is an open source Modelica based modeling and simulation tool designed for industrial and academic usage. Using the Python API for OpenModelica, simulations of the observer are carried out in Python.

Three cases are presented in this section for the dynamic simulations of the developed reduced order nonlinear observer: 
Table 1: Summary of the reduced order nonlinear observer for the hydropower system.

\begin{tabular}{|c|c|}
\hline Plant & $\begin{array}{l}\frac{d x_{1}}{d t}=\frac{Z_{\mathrm{p}}}{\rho}\left(p_{\mathrm{n}}-p_{\mathrm{tr} 1}\right)+A_{\mathrm{p}} g \frac{H_{\mathrm{p}}}{L_{\mathrm{p}}}- \\
\frac{1}{8} \pi f_{\mathrm{D}} B_{\mathrm{p}} x_{1}\left|x_{1}\right| \\
\frac{d x_{2}}{d t}=\frac{Z_{\mathrm{s}}}{\rho}\left(p_{\mathrm{n}}-p^{\mathrm{atm}}\right)-A_{\mathrm{s}} g \frac{H_{\mathrm{s}}}{L_{\mathrm{s}}}- \\
\frac{1}{8} \pi f_{\mathrm{D}} B_{\mathrm{s}} x_{2}\left|x_{2}\right| \\
\frac{d x_{3}}{d t}=\frac{x_{2} H_{\mathrm{s}}}{A_{\mathrm{s}} L_{\mathrm{s}}}\end{array}$ \\
\hline Observer & $\begin{array}{l}\hat{x}_{2}=\hat{\zeta}_{2}-L_{1} x_{1}-L_{3} x_{3} \\
\frac{d \hat{\zeta}_{2}}{d t}=\frac{Z_{\mathrm{s}}}{\rho}\left(p_{\mathrm{n}}-p^{\mathrm{atm}}\right)-A_{\mathrm{s}} g \frac{H_{\mathrm{s}}}{L_{\mathrm{s}}}- \\
\frac{1}{8} \pi f_{\mathrm{D}, \mathrm{s}} B_{\mathrm{s}} \hat{x}_{2}\left|\hat{x}_{2}\right|+L_{3}\left(\frac{\hat{x}_{2} H_{\mathrm{s}}}{A_{\mathrm{s}} L_{\mathrm{s}}}\right)+ \\
L_{1}\left(\frac{Z_{\mathrm{p}}}{\rho}\left(p_{\mathrm{n}}-p_{\mathrm{tr} 1}\right)+A_{\mathrm{p}} g \frac{H_{\mathrm{p}}}{L_{\mathrm{p}}}-\right. \\
\left.\frac{1}{8} \pi f_{\mathrm{D}, \mathrm{p}} B_{\mathrm{p}} x_{1}\left|x_{1}\right|\right)\end{array}$ \\
\hline $\begin{array}{l}\text { Design } \\
\text { variables }\end{array}$ & $\begin{array}{l}\frac{Z_{\mathrm{s}}}{K_{\mathrm{pdz}}}-\frac{1}{K_{\mathrm{pdz}} K_{p}} \leq L_{1} \leq \frac{Z_{\mathrm{s}}}{K_{\mathrm{pdz}}} \\
L_{3} \leq 0\end{array}$ \\
\hline
\end{tabular}

- Only the penstock volumetric flow rate $\dot{V}_{\mathrm{p}} \approx 19.07$ $\left(x_{1}\right)$ is injected, i.e., $L_{3}=0$.

- Only the surge tank water height $h_{\mathrm{s}}\left(x_{3}\right)$ is injected, i.e., $L_{1}=0$.

- Both the penstock volumetric flow rate and the surge tank water height $\left(x_{1}\right.$ and $\left.x_{3}\right)$ are injected.

Measurement data from a real hydropower plant are not available, instead outputs from hydropower model simulations are used. Two hydropower models are used to represent reality: (a) a detailed model, and (b) the simplified model as described in Section 3. Hence, for all cases below, the hydropower models are first simulated separately to get the appropriate synthetic measurements.

A set of dynamic simulations for the reduced order nonlinear observer is performed, where the simulations start from the steady state $\left(\dot{V}_{\mathrm{p}} \approx 19.07 \mathrm{~m}^{3} / \mathrm{s}\right.$ and $h_{\mathrm{s}} \approx 69.91 \mathrm{~m}$ ) and last for $60 \mathrm{~s}$ with a sample time of $0.5 \mathrm{~s}$. A disturbance occurs at time $10 \mathrm{~s}$ with a rapid closing of the turbine valve by $3 \%$. First, an appropriate synthetic measurement data for the penstock flow rate and for the surge tank water height are created from the simulation of the simplified hydropower model. These measurements are shown in Fig. 2.

Moreover, the effect of measurement noise on the estimated state is also of interest. White measurement noise, $v_{k}$, is added to the measurement signals and used for the observer simulations. For both measurements, the mean of the measurement noise $v_{k}$ is zero, i.e., $\bar{v}_{k}=$ 0 , and the noise covariance $V_{k}$ are the same for both measurements for simplicity and equals $V_{k}=0.0001$, i.e., $v_{k} \sim \mathcal{N}(0,0001)$. The noisy measurements are also shown in Fig. 2.
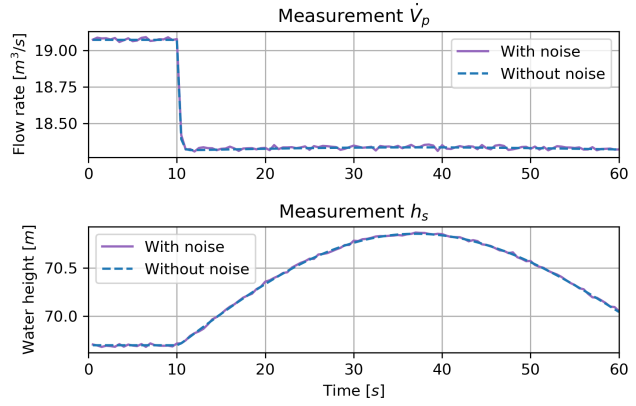

Figure 2: Measurements from the simplified hydropower model with and without noise for the dynamic simulations.

\subsection{Using one measurement}

First, the studies of the observer behavior that uses only one measurement (one state is injected) are performed, i.e., one of the observers' gains is set to zero. The results of the dynamic simulations without any measurement noises for these cases are shown in Fig. 3 for the observer that use only the penstock flow rate measurement $\left(L_{3}=0\right)$ and in Fig. 5 for the observer that use only the surge tank water height measurement $\left(L_{1}=0\right)$. In both figures, three values for the nonzero observer gain are used to study the observer behavior.

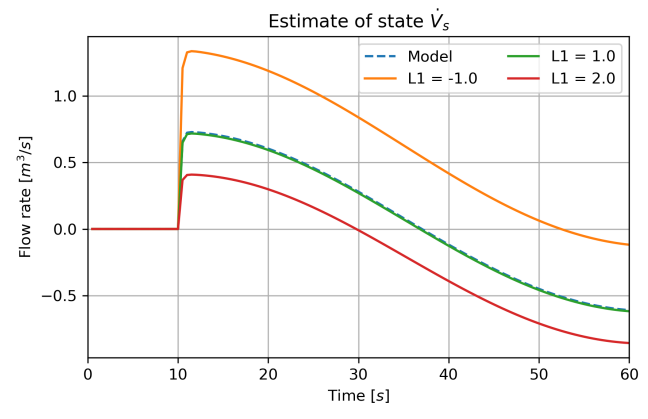

Figure 3: Observer behavior when only measurement $x_{1}$ is injected. Gain $L_{1}$ is varied to study its effect on convergence. 


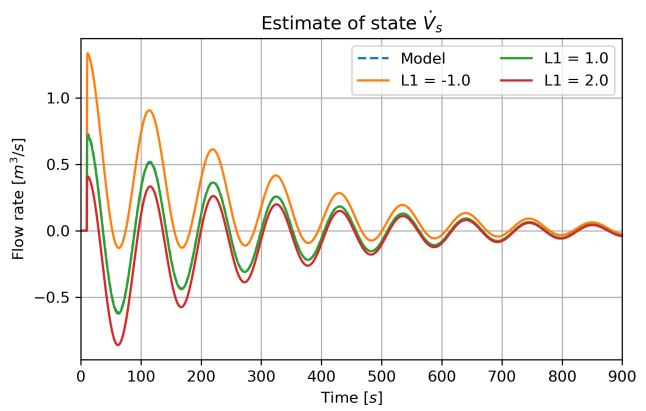

Figure 4: Observer behavior for longer simulation time when only measurement $x_{1}$ is injected.

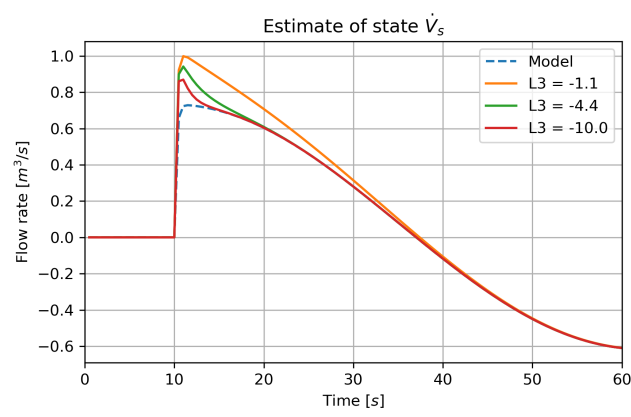

Figure 5: Observer behavior when only measurement $x_{3}$ is injected. Gain $L_{3}$ is varied to study its effect on convergence.

From Fig. 3, it is seen that the estimates from the observer that uses only the penstock flow rate measurement as injection converges properly for the gain value of $L_{1}=1.0$. With the values of observer gain as $L_{1}=-1.0$ and $L_{1}=2.0$, significant overshooting and undershooting are seen during the transient period, respectively. With these values for the gain $L_{1}$, the observer requires a relatively longer time for convergence, see Fig. 4 with extended simulation time of $900 \mathrm{~s}$. Thus a proper choice of observer gain $L_{1}$ seems to be necessary for obtaining faster convergence.

Figure 5 shows that when only the surge tank water height measurement is used as injection, the observer shows some overshooting in the estimates when the disturbance occurs, and then converges to the correct value. Here, a lower value of the observer gain $L_{3}$ leads to faster convergence.

From both these cases, it can be concluded that it is possible to estimate the unmeasured state $x_{2}$ by using only one of the available measurements. However, it is also of interest to check the observer's performance when both measurements are used together.

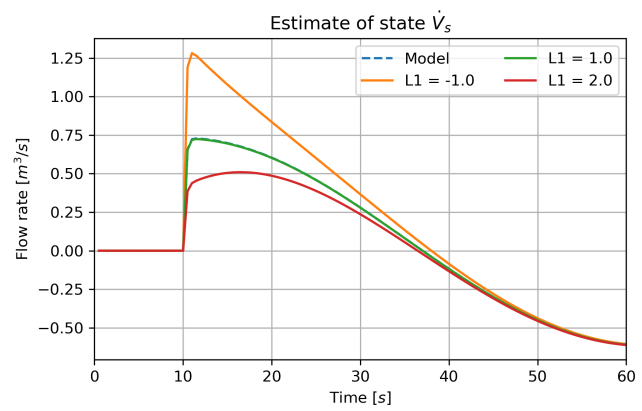

Figure 6: Observer behavior when both measurement $x_{1}$ and $x_{3}$ are injected. Gain $L_{1}$ is varied to study it's effect on convergence.

\subsection{Using both measurements}

The next set of simulations show the behavior of the observer that uses both the penstock flow rate and the surge tank water height as available measurements. First, dynamic simulations for the reduced order nonlinear observer without any measurement noises are presented in Fig. 6. Here, the observer gain $L_{1}$ is varied and another gain is set to a fixed value of $L_{3}=-1.0$.

As compared to Fig. 3 (where only one measurement was injected), Fig. 6 shows that by using both the available measurements, the performance of the observer is significantly improved. With the same choices of gain $L_{1}$ (for both cases), it can be seen that the observer converges much faster when both measurements are used in the observer design. It is also noticed but not shown in Fig. 6 that with lower values for the observer gain $L_{3}$, the estimates converges relatively faster to their correct values.

However, the observer with the fixed gain $L_{1}=1.0$ and varying gain $L_{3}$ produces very similar results for different choices of gain $L_{3}$. This is shown in Fig. 7, where measurements without noises are used for dynamic simulation of the reduced order nonlinear observer. Here, different values are used for the observer gain $L_{3}$ and the other gain is set to a fixed value of $L_{1}=1.0$.

As compared to Fig. 5 (where only one measurement was used), Fig. 7 shows that the performance of the observer is vastly improved when both measurements are used. In this case, the overshoots are reduced significantly. It can be concluded that when both measurements are used in the observer design, and with proper choices of gains $L_{1}$ and $L_{3}$, the performance of the observer can be vastly improved. However, it is also possible to estimate the unmeasured state by using only one measurement. With this, the proof of convergence of the observer design perhaps will also be simplified. 


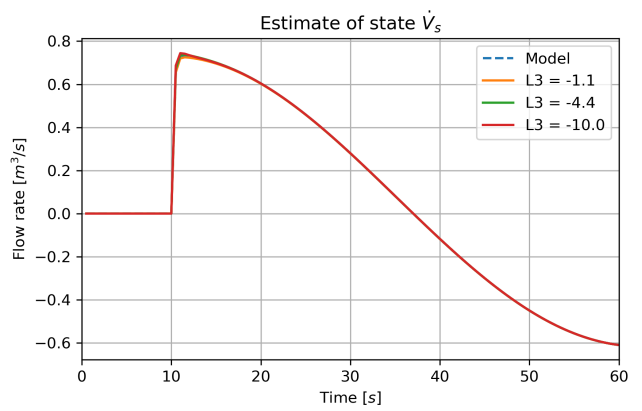

Figure 7: Observer behavior when both measurement $x_{1}$ and $x_{3}$ are injected. Gain $L_{3}$ is varied to study it's effect on convergence.

In addition, in some cases it might be considered to use a simple observer for estimating $x_{2}$ (the surge tank flow rate) that is based only on Eq. 4 and use changes in $x_{3}$ (the surge tank water height) as measurements (through model inversion). This simple observer will be more sensitive to noise, but otherwise it would have many advantages, such as simplicity (no tuning parameters) and ease of implementation. However, this simple observer will work fine as long as the model is considered to be perfect. In reality, model parameters might not be exactly known and/or there might be other unknown input disturbances acting on the system. Under such conditions, open-loop observers may not function well and it might be necessary to introduce feedback by injecting the measurements. Furthermore in Fig. 7, it has been shown that by using measurement $x_{1}$ in addition to $x_{3}$ as the measurement, the estimation can be significantly improved.

\subsection{Measurement noise influence}

The performance of the observer is affected by the measurement noise and the choice of the observer gains. To illustrate this, the observer gain $L_{3}$ is varied while the gain $L_{1}$ is kept constant. The influence of the measurement noise on the estimate is shown in Fig. 8.

As the gain $L_{3}$ is lowered, the observer converges faster, however, this also makes the estimates more noisy. This justifies the fact that under the presence of measurement noises, the speed of convergence and the occurrence of noisy estimates should be balanced properly by proper choice of observer gains. To increase the performance of the observer with noisy measurements, the measurement data should probably be preprocessed and filtered before feeding it to the observer in practise.

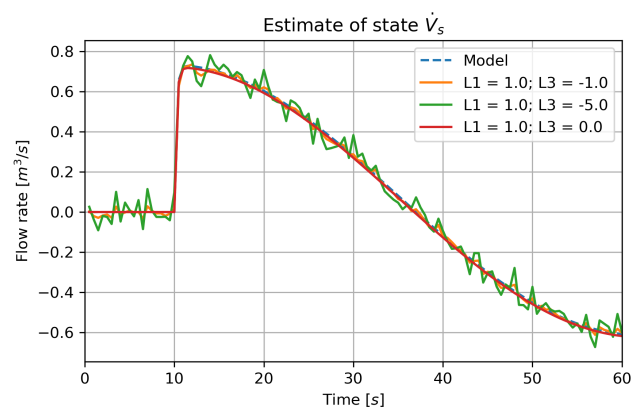

Figure 8: Effect of measurement noise on the state estimates with varying gain.

\section{Testing observer with measurement data from a detailed model}

It is of interest to see how the reduced order nonlinear observer behave when the synthetic measurements are based on a more detailed/realistic model while the simple model is used for the design of the observer. The detailed model used for the synthetic measurements is similar to the model presented above, but includes water compressibility and pipe elasticity in the penstock, see Vytvytskyi and Lie (2017) for more information about this model. Moreover, the Darcy friction factor varies with the flow rate for all pipe units in this detailed model.

First, appropriate synthetic measurements for the penstock flow rate and for the surge tank water height are created from the simulation of the more detailed hydropower model. These measurements are shown in Fig. 9 for the cases without and with measurement noise. Similarly to the previous case, the same level of measurement noise $v_{k}$ is used here.
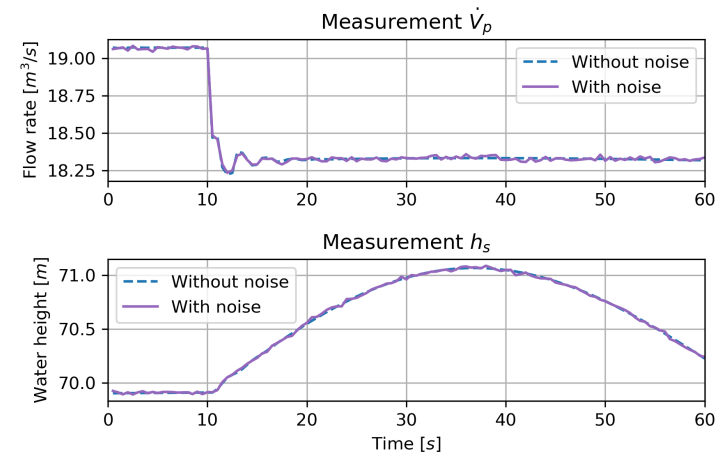

Figure 9: Measurements from the more detailed hydropower model. 
The dynamic simulations for the reduced order nonlinear observer that use these measurements from the detailed model without considering measurement noise are shown in Fig. 10. Here, the results of the observer with re-tuned gains $L_{1}$ and $L_{3}$ that provides relatively good estimation is shown in the figure.

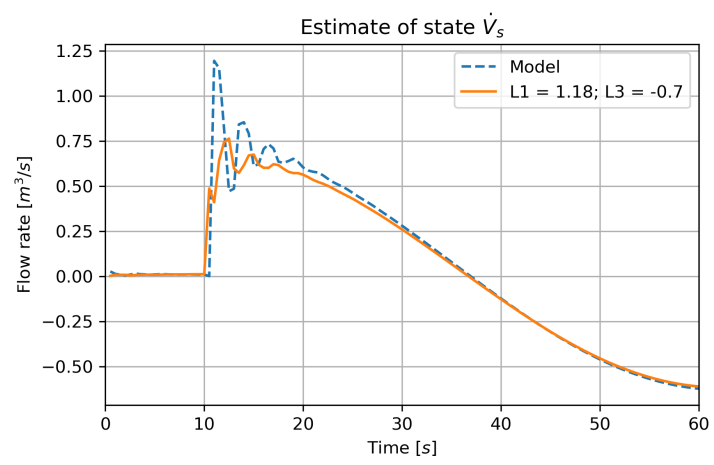

Figure 10: Observer behavior when both measurement $x_{1}$ and $x_{3}$ are injected. Measurements without noise from the more detailed hydropower model.

Figure 10 shows that fine tuning of the $L_{1}$ and $L_{3}$ observer gains reduce the offset between the estimates from the observer and the results from the detailed hydropower model.

Simulations with noisy measurements from the detailed hydropower model are performed to see the influence of the noise on the estimation. The results from the observer simulation are shown in Fig. 11, where it can be seen that the measurement noise affects the observer results, and the estimates from the observer become noisier. This behavior is expected and is similar to the results from the previous case study (Fig. 8). It is also clearly visible that observer produces relatively good estimates even under the presence of measurement noises from the detailed hydropower model.

It is worth mentioning that the observer gains had to be re-tuned when it is applied on a detail model. In both cases (with/without considering measurement noises), it can be seen that the observer converge to the true state values. However, the performance of the observer is relatively poor compared to the case where perfect model (no model-observer mismatch) is considered. Under the presence of measurement noises, the estimates also become slightly noisy as shown in Fig 11.

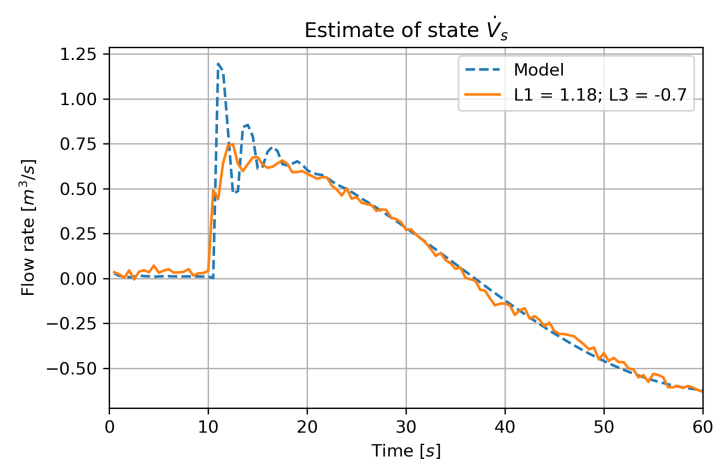

Figure 11: Observer behavior when both measurement $x_{1}$ and $x_{3}$ are injected. Measurements with noise from the more detailed hydropower model.

\section{Conclusions}

The design and proof of convergence for the reduced order nonlinear observer for the simplified hydropower system has been presented in this paper. This observer is designed to estimate the surge tank flow rate using the measurements from two other states (the penstock flow rate and the surge tank water height). The observer has been implemented in OpenModelica and added to our in-house hydropower library (OpenHPL). Then a number of simulations have been run in Python using the Python API for OpenModelica in order to study the designed observer.

The dynamic simulations have been performed to show the observer behavior under the presence of input disturbance. These simulations have been carried out for three cases, where the measured states have been injected separately first, and then both measured states have been used. Moreover, the influence of the measurement noise on the observer has also been presented for the dynamic simulations. The simulations showed that the performance of the reduced order nonlinear observer mostly depends on the observer gain $L_{1}$ for overshooting/undershooting of the estimates, and the gain $L_{3}$ for the convergence speed.

It has also been checked, but not shown in the paper, how the observer behaves if the designed conditions for the observer gains are broken, e.g., $L_{3}>0$ or $L_{1} \leq$ $\frac{Z_{\mathrm{s}}}{K_{\mathrm{pdz}}}-\frac{1}{K_{\mathrm{pdz}} K_{p}}$ and $L_{1} \geq \frac{Z_{\mathrm{s}}}{K_{\mathrm{pdz}}}$. In case with $L_{3}>0$, the system becomes unstable and the observer fails. For another case, with $L_{1}$, the simulation runs without failures. However the results become poorer.

To summarize, it has been shown that a reduced order nonlinear observer can be designed and used for the state estimation in the hydropower system. The design of the observer is based on a simplified model with cer- 
tain assumptions. This lowers the complexity in the design of the observer. When the observer is tested against the simplified model, the estimates are proper without any offsets. However, the observer is also tested against the data from a more detailed/complex hydropower model where simplifying assumptions are not considered. Under such condition, the observer has to be re-tuned. With a properly calibrated/tuned reduced order observer, the observer can still provide satisfactory estimates of the unmeasured state.

\section{References}

Anderson, B. D. O. and Moore, J. B. Optimal Filtering. Prentice-Hall, Englewood Cliffs, NJ, 1979.

Andrieu, V. and Praly, L. On the Existence of a Kazantzis-Kravaris/Luenberger Observer. SIAM J. Control and Optimization, 2006. 45(2):432-456. doi:10.1137/040617066.

El-Farra, N., Mhaskar, P., and Christofides, P. D. Output feedback control of switched nonlinear systems using multiple Lyapunov functions. Proceedings of the 2005, American Control Conference, 2005., 2005. 6:3792-3799.

Kravaris, C., Sotiropoulos, V., Georgiou, C., Kazantzis, N., Xiao, M., and Krener, A. J. Nonlinear observer design for state and disturbance estimation. Systems \& Control Letters, 2007. 56(11):730 - 735. doi:10.1016/j.sysconle.2007.05.001.

Krener, A. J. and Isidori, A. Linearization by output injection and nonlinear observers. Systems \& Control Letters, 1983. 3(1):47 - 52. doi:10.1016/01676911(83)90037-3.

Lie, B., Bajracharya, S., Mengist, A., Buffoni, L., Kumar, A., Sjölund, M., Asghar, A., Pop, A., and Fritzson, P. API for Accessing OpenModelica Models From Python. In Proceedings of the EuroSim 2016, Oulu, Finland. 2016.
Luenberger, D. G. Observing the State of a Linear System. IEEE Transactions on Military Electronics, 1964. 8:74-80. doi:10.1109/TME.1964.4323124.

Simon, D. Optimal State Estimation: Kalman, $H \infty$, and Nonlinear Approaches. John Wiley \& Sons, 2006.

Smith, M. C. The general problem of the stability of motion: Translated and Edited by A. T. Fuller. Taylor and Francis, 1992. Automatica, 1995. 31:353354. doi:10.1016/0005-1098(95)90022-5.

Splavska, V., Vytvytskyi, L., and Lie, B. Hydropower Systems: Comparison of Mechanistic and Table Look-up Turbine Models. In Proceedings of the 58th Conference on Simulation and Modelling (SIMS 58) Reykjavik, Iceland, September 25th27th, 2017, 138. Linköping University Electronic Press, Linköpings Universitet, pages 368-373, 2017. doi:10.3384/ecp17138368.

Vytvytskyi, L. and Lie, B. Comparison of elastic vs. inelastic penstock model using OpenModelica. In Proceedings of the 58th Conference on Simulation and Modelling (SIMS 58) Reykjavik, Iceland, September 25th-27th, 2017, 138. Linköping University Electronic Press, Linköpings Universitet, pages 20-28, 2017. doi:10.3384/ecp1713820.

Vytvytskyi, L. and Lie, B. Linearization for Analysis of a Hydropower Model using Python API for OpenModelica. In Proceedings of the 59th Conference on Simulation and Modelling (SIMS 59) Oslo, Norway, September 26th-28th, 2018, 153. Linköping University Electronic Press, Linköpings Universitet, pages 216-221, 2018a. doi:10.3384/ecp18153216.

Vytvytskyi, L. and Lie, B. Mechanistic model for Francis turbines in OpenModelica. IFACPapersOnLine, 2018b. 51(2):103 - 108. doi:10.1016/j.ifacol.2018.03.018. 